\title{
RPL for accreditation in higher education: as a process of mutual understanding or merely lifeworld colonisation?
}

\author{
Fredrik Sandberg and Per Andersson
}

\section{Linköping University Post Print}

N.B.: When citing this work, cite the original article.

This is an electronic version of an article published in:

Fredrik Sandberg and Per Andersson, RPL for accreditation in higher education: as a process of mutual understanding or merely lifeworld colonisation?, 2011, Assessment \&amp; Evaluation in Higher Education, (36), 7, 767-780.

Assessment \&amp; Evaluation in Higher Education is available online at informaworldTM:

http://dx.doi.org/10.1080/02602938.2010.488793

Copyright: Taylor \& Francis (Routledge)

http://www.routledge.com/

Postprint available at: Linköping University Electronic Press

http://urn.kb.se/resolve?urn=urn:nbn:se:liu:diva-63348 


\title{
RPL for accreditation in higher education - As a process of mutual understanding or merely lifeworld colonisation?
}

\author{
Fredrik Sandberg ${ }^{1}$ \\ Department of Behavioural Sciences and Learning, Linköping University, Linköping, \\ Sweden
}

\author{
Per Andersson \\ Department of Behavioural Sciences and Learning, Linköping University, Linköping, \\ Sweden
}

This article focuses on a process of recognition of prior learning (RPL) in higher education. It is based on experiences from a project carried out in collaboration between the University of Lund, Linköping University and two trade unions in Sweden. The aim of the project was to find ways of recognising prior learning for accreditation of course credits at university level. In the project and its analysis Habermas' theory of communicative action was used as theoretical underpinning. During the project we carried out a thematic analysis based on interviews. The analysis suggested that the participants had a mystified view of higher education, that RPL must be considered a learning process and that the participants prior learning can be characterised as practical wisdom. These themes guided the development of the project. In a retrospective analysis, language proficiency seemed to play a significant role for the outcome of the process. The conclusion suggests that a more fair and valid assessment of the participant's prior learning could be accomplished by focusing on RPL as a process of mutual understanding. However, a critical appraisal of the process suggests that RPL for accreditation is problematic and could be seen as a colonisation of the lifeworld.

Keywords: Recognition of prior learning; accreditation; mutual understanding; Habermas; higher education; colonisation

\section{Introduction}

This article discusses an RPL initiative targeting employees in two Swedish trade unions. The possibility of giving recognition to their working life experience and competence, in terms of credits in initial courses in higher education was explored in the project Validus. Recognition or assessment of prior learning (RPL, APL) is an aspect of many educational practices today. It seems that more and more attention is being paid to the idea that pupils and students have learnt something before entering (formal) education, and that they learn things outside the educational setting. This interest in RPL is related to the focus on both lifelong and lifewide learning. This means that learning is an ongoing process (lifelong), at the same time as learning

\footnotetext{
${ }^{1}$ Linköping University, Department of Behavioural Sciences and Learning, 58183 Linköping. Sweden. Email: fredrik.sandberg@liu.se
} 
takes place in different contexts such as leisure, work and education (lifewide). Consequently, the interest in RPL is particularly large in adult learning contexts such as adult education, vocational (working life) learning, and higher education. It should be noted that in the present study we use the abbreviation RPL and 'recognition' as a broad concept including more formal assessment processes also named 'accreditation of prior (experiential) learning' (APEL).

A central aspect of RPL is the relation to some sort of transfer of knowledge or competence. Knowledge is assessed to make it easier to get recognition for it in a new context. It could, for example, be a matter of transfer between countries, between different educational systems, or from a context of informal learning to a context of formal learning. The latter is the case when the results of informal learning in working life or in everyday life are assessed and accorded recognition in relation to the demands of the educational system. But RPL can also be seen as a tool to transfer individuals. For instance, RPL for access can be seen as a method of transferring individuals into higher education by enabling broader participation. For example, in South Africa RPL has been used as such a tool for the transfer of individuals (Castle and Attwood 2001).

The project studied in the present article was carried out in collaboration between the University of Lund, Linköping University and the trade unions The Swedish Municipal Workers' Union and The Union of Civil Servants. The project was organised in two phases. The purpose of the first phase was to create a model and methods for an RPL process. Nineteen interviews were conducted with the purpose of finding out what kind of knowledge the elected representatives had, and the interview method use here was also tested as a possible method to be used in the RPL model. In phase two, the aim was to assess and test the model and methods developed on individuals working in the trade unions. The purpose of this article is to report on this project, focussing on aspects of fairness and validity, and critically appraise some aspects of RPL for accreditation. The main questions are: How can we support fairness and enhance the validity in RPL for accreditation? What are the main problems with RPL for accreditation in higher education seen through the lens of Habermasian theory?

We used thematic analysis to discover important themes during the progression of the project as well as in the retrospective analysis conducted after the project had ended. Jürgen Habermas' theory of communicative action (TCA) $(1984 ; 1987)$ was used as theoretical input, starting from the central concepts of system and lifeworld, and the potential colonisation of the lifeworld by the system, a process that could result in a dominance of an instrumental rather than communicative rationality. In RPL, practitioners deal with assessing and transferring individuals' prior experiences to other contexts. Individual experiences from work, leisure, etc. are often formalised and taken up by different systems (i.e. the higher education system). Here, Habermas' TCA contributes by focusing on communication and providing the underlying conditions for how individuals can reach mutual understanding in a process signified by communicative rationality. This should be a main concern, since prior learning cannot be assessed if the individual is not able to put his/her learning into an understandable context for assessment, e.g. into words. Further, the assessors must be able to make higher education understandable, since participants in RPL may have no experience of this context.

On the other hand, Habermas is critical of how different kinds of systems (for instance, the economy and bureaucracies) in modern society seem to neglect, (or, as Habermas puts it, colonise) human experiences. RPL could be seen as a rather 
instrumental process where knowledge becomes a medium like money used in exchange for a formal grade. However, RPL is a way of confirming knowledge, which, if not confirmed, may have remained unnoticed. In this article we discuss an RPL project aimed at providing accreditation in higher education courses. Each course has specific criteria, which have to be met. This means that the system demands specific knowledge from the participants. Even though this is a rather instrumental and rigid process, the aim of this article is to provide ideas about how this process to some extent can be made valid and fair. The focus is on how assessors need to keep in mind that the participants often lack experience of higher education and do not fully know how to express their prior learning so that it fits this context. In this article, it is argued that Habermas could contribute a theoretical underpinning to:

1. Provide tools for more critical reflection on the nature of RPL in higher education for accreditation

2. Focus on mutual understanding as a tool to support fairness and enhance validity in RPL for accreditation.

\section{Research on recognition of prior learning}

RPL is a research area that is being developed. The current 're-theorisation' of RPL is presented by Andersson and Harris (2006), who bring together analyses of RPL from a number of different perspectives: assessment theory, the sociology of education, post structuralism and situated knowledge/learning theory, activity, actor-network and complexity theory, and symbolic interactionism. However, there is a lack of more critical research on RPL in higher education.

This article focuses in particular on RPL for accreditation in a higher education context. A central theme, which has been discussed when it comes to RPL in this context, is the extent to which RPL is possible. For example, on the one hand Chisholm and Davis (2007) argue that a retrospective and reflective portfolio model could result in $100 \%$ accreditation for e.g. a professional doctorate, similar to the model of awarding a Ph.D. based on previously published work. On the other hand, Wheelahan (2006) promotes RPL as an integrated part of all qualifications, but not as a technique for validating a complete degree. She sees this integration of RPL and education as necessary in the development of 'graduateness' - the point being that you cannot become a graduate merely from informal learning, but the formal educational process contributes something more that is essential; for example development of the communicative aspect of individual competence.

Castle and Attwood (2001) expand the criticism of RPL for accreditation in higher education even further. They argue that RPL for accreditation in higher education is in itself problematic. Drawing on experiences of RPL in higher education in South Africa, they conclude that RPL should be targeted at access, rather than accreditation. In a recent article, Stenlund (2009) has also criticised RPL/APL from an assessment perspective, based on the lack of both theoretical and empirical research of validation of RPL models. Using critical discourse analysis, Peters (2005) shows how control and exclusion can be the result when the RPL discourse is something candidates have to learn, rather than something that acknowledges what they already know.

Pokorny (2006) uses actor network theory to conclude that instead of focusing solely on the convergence of candidates' knowledge with academic networks; we could also focus on the heterogeneity of their knowledge networks. The latter would probably make RPL more open to learning from outside academia. Starr-Glass (2002) 
argues that a focus on predictive validity in the assessment of prior learning, instead of concurrent validity, would place the focus on future potential in academia and working life, instead of on equivalency with a current definition of a subject area. $\mathrm{He}$ also maintains that a broader range of competence could be made visible if we begin the assessment from the language and experiences of the candidate. Further, StarrGlass and Schwartzbaum (2003) analyse the problems and possibilities in accreditation of prior learning in Judaic studies. They see how accreditation deals with values and examinations that are located in different structures of learning (in their case, 'sacred' and 'secular' structures). They argue that assessors and assessees, and representatives of the different structures, could meet in the 'liminal space' between these structures to possibly develop more creative recognition processes.

In this article, one main issue is the very concept of RPL for accreditation, also critically scrutinised by Castle and Attwood (2001). To what extent is such accreditation possible and what consequences does this concept of RPL have? Here, it is important to emphasise Wheelahan's discussion on graduateness as it addresses an important aspect of the outcome of learning in higher education - an aspect which accreditation through RPL may not be able to produce. But, even though there are problems with RPL for accreditation, the idea of a 'liminal space' put forward by Starr-Glass and Schwartzbaum (2003) could be a way of further developing such processes. For example, Valk (2009), in her overview of RPL in European higher education, describes how RPL (or APEL) in higher education in the UK could take place in official university courses where candidates reflect on, identify and give proof of their prior learning, and an analysis of a teacher education initiative in South Africa (Volbrecht 2009) identifies a high degree of 'Trojan Horse RPL' (Harris 1999) where the location of RPL in the curriculum brings new groups into the university. In this article, processes of mutual understanding could be seen as something that happens in this type of 'liminal space'. It is proposed that this could support fairness and strengthen the validity of RPL for accreditation, even though, this article is critical of the very nature of such RPL processes.

\section{A Habermasian perspective}

In the analysis of the Validus project and RPL, we use some concepts from Habermas' theory of communicative action (TCA). We especially focus on the concepts of system and lifeworld, and instrumental and communicative rationality.

Two main concepts in Habermas' TCA are system and lifeworld (Habermas 1984; 1987). Subsystems such as the economy and bureaucracies play a major role in an increasingly complex society. The key media for integration and reproduction in these systems are money (economy) and power (bureaucracies).

The lifeworld can be defined as a horizon of experiences. In, for instance, education and family, language plays a significant role in socialisation and the creation of identities. For example, we could talk about the lifeworld of education when referring to such elements in education as mutual learning processes, discussions and communication in general. In the lifeworld, language is the medium for integration. One important distinction is that between Habermas' lifeworld and the more common understanding arising from a phenomenological point of view (see e.g. Husserl 1970). For Habermas, the lifeworld is a horizon of experiences, changed and reproduced through collective communication. In our family, in education and so forth the lifeworld is the unquestionable background of experiences which are always 
there when we communicate. In phenomenology, as suggested by Husserl, the lifeworld precedes all knowledge - and is found and looked for in the subjective experiences of the individual. For Husserl research should try, as much as possible, to find the experiences grounded in the lifeworld. Habermas, on the other hand focuses on the possibilities for the lifeworld to be reproduced through inter-subjective learning processes. Thus, one main difference between the phenomenological lifeworld, as suggested by Husserl and the Habermasian is that, whilst Husserl focuses on the subjective, Habermas' focal point is the inter-subjective dimension.

Habermas' point is that systems in modern society act more and more instrumentally and self-regulating. In this process, the lifeworld, our shared horizon of experiences, is under threat of colonisation. This primarily means that the possibility for individuals to communicate, discuss and reach a consensus becomes more and more limited. George Orwell's '1984' (1950) could be used as an extreme example of a society where the lifeworld is totally colonised and society is reproduced as a system. The result could then be that experiences grounded in the lifeworld will not be reproduced (or only reproduced when fitting the demands of the system). Instead, society is reproduced by means of steering media such as money and power. In this scenario, the symbolic reproduction of the lifeworld cannot be achieved since other media (money and power) are used for integration instead of language. Society is then reproduced as a system.

Habermas connects two forms of rationality to the concepts of system and lifeworld. The system works through an instrumental rationality where money and power serve as the primary media for integration. To be able to change or reproduce the system, money and power must always be taken into consideration while language becomes a subordinated medium. When instrumental rationality takes over in contexts such as education, the symbolic reproduction of the lifeworld is threatened. This means that learning is no longer a matter of discussions between individuals aiming for mutual learning and understanding. It could instead promote individualistic and strategic actions for gaining grades. However, on the other hand Habermas talks about communicative rationality, which forms the basis of communicative action. It is here suggested that when language is used as integrating medium and the underlying conditions is acceptable individuals can reach mutual understanding. Habermas points out that in communicative action: 'participating actors must conduct themselves cooperatively and attempt to reach an agreement about their plans [. . .] on the basis of common situation interpretations' (Habermas 1992, p. 79).

Habermas' $(1984,1987)$ TCA focuses on the rationalisation and thus reproduction of the lifeworld. This rationalisation means that language acquires a central function in reaching understanding, co-ordinating action and socialising individuals. Language is a medium of cultural reproduction, social integration, and socialisation. In communicative action, co-ordinating action through understanding is central. A prerequisite for understanding is communicative rationality, which have several dimensions. It is characterised not only by a means-ends rationality, but also by normative and expressive rationality. In other words, purposeful action is not enough - in addition you have to act according to valid norms and rules, and to give a truthful expression or presentation of the self. These three dimensions of rationality form the basis for reaching mutual understanding in a process characterised by, as mentioned before, communicative rationality.

The importance of communicative rationality, communication and mutual understanding can be seen in education, since it has a central function in the reproduction of the lifeworld. Nevertheless, educational processes are not only 
characterised by communicative rationality and mutual understanding. A central aspect of most formal educational processes also involves the assessment of students' knowledge by means of tests, grades, etc. Extensive research (see e.g. Kirkland 1971; Madaus 1988; Dahlgren et al. 2009) shows how assessments are part of the 'hidden curriculum' (Snyder, 1970), for example, making students act as 'cue-seekers' (Miller and Parlett 1974). The hidden curriculum can be seen as a consequence of a lack of mutual understanding and communicative rationality. 'Cue-seeking' also relates to consequences that arise when grades and tests become the main target of education. When individuals are not given the chance to engage in mutual learning processes, there is a risk that students become individualistic cue-seekers trying to figure out what is needed to succeed in tests and get high grades.

In this article, we on the one hand argue for the importance of mutual understanding in RPL processes to support a fairer and more valid assessment. On the other hand, a critical part of the article, based on Habermas, addresses some more general concerns regarding RPL for accreditation, drawing from the specific context of RPL for accreditation presented in this article.

\section{Methodological considerations}

The results reported in this article are based on documents, observations, interviews and more informal conversations with participants during the Validus project. The results from the project were also presented in two reports (Sandberg and Schultz 2007; Schultz and Sandberg 2007). These reports act as a background and can be seen as 'empirical' documents in our analysis. The analysis has mainly been performed after the project and we employed the Habermasian perspective to discuss and scrutinise the project. One of the authors of the present article (Sandberg) was deeply involved in the project work, while the other (Andersson) was only marginally involved. Consequently, we were able to analyse the content and results of the project from both an insider and relative outsider perspective. Our analysis is thus based on these insider and outsider understandings, our pre-understanding of the phenomenon RPL, and the theoretical pre-understanding based on Habermasian ideas. In a process of hermeneutic interpretation, these different horizons of understanding have interacted and contributed to the development of a new understanding of some aspects of RPL (cf. Warnke 1987).

Project Validus was a practical project for developing an RPL model for accreditation of knowledge for credit points in higher education, and not a theoretical research project. Despite this being the case, early in the project, Habermasian theory seemed to be an interesting perspective. For instance, we acknowledged that it was of great importance to understand each other in the contexts of working life and higher education.

\section{RPL in the Validus Project}

In this section, we will first present the Validus RPL model and three central themes that emerged during, and guided, the project work. Secondly, we will analyse the project retrospectively in relation to our theoretical perspective.

To start with, the project ended up in an RPL model including the following steps:

1. Information about the RPL process 
2. Questionnaire

3. Course selection and self-evaluation of formal credentials

4. Mutual conversations

5. Case

6. Conclusion and assessment

7. Feedback

8. Completion of the course

In the second phase of the project, a potential model was tested on 12 participants. Except for one male, the participants were women (on average middle-aged). The participants from the Swedish Municipal Workers' Union (10 in all) had a background as care workers (elderly care, children's care); while the participants from The Union of Civil Servants had a background of working with more academically qualified jobs ( 2 in all). The model focused on recognising these participants' prior learning in the framework of three courses at Linköping University. The courses were initial courses at the undergraduate level in the social sciences (with focus on sociology): 1. The individual and the working team. 2. Multiculture in society and work-life. 3. Managing and organising health and welfare systems. Each course gave 7.5 ECTS points.

Information was considered highly important in order to make the process transparent. Accordingly, all the participants were given an information folder containing a brief introduction to RPL. The whole RPL process was also introduced step by step with each step containing information and instructions. The purpose was to make the RPL process easy to understand.

In the second step, the participants answered a questionnaire containing questions about their prior knowledge and experience of higher education, why they wanted to be part of an RPL process and their hopes for the outcome of the process. The purpose of the questionnaire was to gain knowledge about the participants' background, check up on their prior experiences from higher education and gain some understanding of their thoughts on what the process could contribute. In step three, the participants selected a course and from the curriculum of the course they were asked to present their existing formal credentials (certificates, grades etc.) with reference to their prior learning. In step four, we focused on conversations in order to reach mutual understanding. All the participants were invited to a face to face discussion about their prior learning in relation to key terms and themes in the course selected. In step five, the participants were given a case based on the curriculum of the selected course. The purpose of the case was to let the participants use their prior learning to discuss key questions and themes that related to the course content. For instance, in one of the courses, the case was based on a description of a company and different problems that can arise in the organisation. The task was to identify such problems and provide possible solutions, as if participants were consultants hired by the company. They were given a week to complete the case. The case was not restricted in any sense and there were no specific guidelines. The assessment was primarily based on the case (step 5). Formal credentials from prior learning were intended to be a part of the assessment, but only a few of the participants managed to provide relevant prior credentials. In the end, the cases turned out to be sufficient to make a fair assessment. Here, it should be noted that the assessments were made by the regular teachers in the selected courses. Accordingly, the main focus of the RPL project was to prepare and introduce the participants to the case. The assessment per se was made in relation to the same criteria as when assessing 'traditional' participants in the courses. The 
purpose of this was to try to create an RPL model where the participants received the same formal grade as traditional students taking the courses. The aim was to incorporate the RPL model into the existing system. After the case had been assessed, the participant received feedback on the case and was then given the opportunity to complete the course. This involved studying the parts for which they had not been given recognition.

The structure of the final model was influenced by a number of themes that emerged in phase one of the project as a result of a more thematic analysis. Three themes helped us in the development of the project and the final model:

- The participants' 'mystification' of higher education

- RPL as a learning process

- Phronesis - practical wisdom among elected representatives

The participants' mystified view of higher education was related to the suggestion that the participants had little or no experience of higher education. Of course, this is often the case with ordinary students as well. But it seemed important to address, since it, in some cases resulted in misunderstandings. For instance, one participant had attended some courses in higher education and now, as a trade union worker, was looking into the possibilities of broadening the competence of the union's members (primarily assistant nurses). She especially underlined the importance of higher education;

“. . . and then I have a passionate interest in municipal workers [members of the union] in the world of higher education; that we should be more educated and that we have the competence to go further. To enter this world [higher education], know this language and then be able to manipulate you as well. But when it comes to studying they [members] draw back".

One of the issues with the mystification was some of the participants' talk of academia as a specific world with a specific language. Even though this is true in one sense, there is also a risk of creating a distance between higher education and working life. There is also a risk of seeing higher education as the ultimate judge of what kind of knowledge counts (or as providing the individual with a language for 'manipulation'). One participant explained this in the following way: "I don't need these credit points but I think it is important that we have real stuff" suggesting that practical experiences are not 'real', while academic knowledge is.

Another part of the mystification addressed issues of truth. In some of the interviews in phase one, higher education was often discussed as the world providing truths. During an interview in phase one, a participant, for instance, said that: "I could give you an answer to that question but it would not be the one true answer you seek".

In the interviews in phase one the mystification did create an unnecessary and somewhat problematic gap between the participants' understanding of higher education and assessors and researchers. There seemed to be a need to orient the participants in the language of higher education. To be able to make a fair assessment of the participants' prior learning, the conditions, goals and ideas of the RPL process had to be mutually agreed upon and mutually defined.

In phase one of the project, we tried out interviews as a possible method to be used in the RPL model. In phase two, interviews were replaced or developed into what we earlier referred to as mutual conversations. Mutual conversations were useful 
exercises for the participants to learn to reflect on their prior learning and knowledge. Here, we saw language as the key medium for integration between assessor and assessee and we intended to work by means of a communicative rationality. It can be argued that they were forced to 'theorise' their practical knowledge. The mutual conversations in phase two thus became important learning processes where the participants were able to put their prior learning into a more academic styled and reflective language.

The interviews suggested that the knowledge of the participants was both diversified and complex. One finding was the participants' 'practical wisdom' or, in Aristotle's words, 'phronesis'. Many years of experience of dealing with people having problems in their work situation had given the participants skills from informal learning processes. The mutual conversations, in the finalised model, became a way of trying to reach these everyday life experiences and trying to convert and compare them to the content of courses. One problem was, however, that the purpose of the model was to give accreditation in connection with a specific curriculum. These experiences thus to some extent slipped through the cracks of the system, since they do not meet the demands of the curriculum.

\section{Academic terms, language proficiency and assessment}

In the section above we especially focused on an explanation of the concept of mystification, a concept which, in many ways, guided the progression of the model. We argued that mutual understanding became important to integrate the participants into the context of higher education. In this section the analysis advances into a retrospective analysis of the project.

An important part of RPL is to transfer or translate prior learning from one context to another. In this process, assessment, the conversion of practical knowledge into academic terms, and the individual's use of language are important themes.

An academic term and what this term means is an interesting theme to explore. The participants in the project regarded academic themes and concepts as somewhat peculiar and part of 'that other world', which they knew little about. What happened early in the project was that the participants believed that academic terms contain a mysterious truth, which they were unable to grasp with their prior learning. Their response was to become 'cue-seekers'. As cue-seekers, the participants tried to solve the mystery of higher education (e.g. academic terms) individually by searching for the right answers. Here, they were left to their own devices and there was a need for us to more clearly inform, define and clarify the context of higher education and the RPL process. However, to seek 'cues' is perhaps not a specific problem for RPL, it is probably a behaviour that many new students in higher education with non-academic background adopt.

Secondly, skills in using academically styled language became an important issue. If a participant had well-developed language proficiency, although perhaps obvious, she/he was also able to present her/his prior learning more accurately. The conclusion to be drawn from this is that language proficiency needs to be dealt with if RPL is to be fair and valid. But it is evidently a problem, since developed language proficiency is a central part of the outcome of learning in higher education (see also 'graduateness'). On the other hand, however, RPL processes can include exercises that enable individuals to learn to reflect on and express their prior learning. Our intention was to use mutual conversations with the participants to enhance their skills in using language to enable their prior learning to become visible and more 
understandable in relation to the curriculum. This was inspired by Habermas (1984, 1987) and our idea was to use mutual understanding as a tool for enhancing fairness and validity in the RPL process and assessment. In RPL, assessment is a central part of the process. In this case, the RPL process was aimed at translating prior learning to be able to assess it against a fixed curriculum. But assessing prior learning can be a fragile and delicate process, especially when assessing against fixed criteria, which may frame the assessment too narrowly. Usually, failing means that you have put too little effort into studying for the examination and the consequence would be to study more and then take the test once again. Failing to satisfy the curriculum would here mean that several years of working experience were not sufficient. Assessing prior learning therefore deviates from assessing traditional students and teachers working with RPL should be aware of and sensitive to these facts. What is central here is that the assessees understand that experience does not necessarily mean that you have the necessary knowledge, in relation to course content. Alternatively, the problem is that you are not yet able to put your prior knowledge into words, which will be addressed in the next section. But, failing could of course also be the result other aspects; i.e. an insufficient assessment process where the assessor lack the proper skills or where the prior learning is not described well enough. However, when RPL processes progresses through mutual understanding this may also enhance the validity and assessment process. When assessor and assessee mutually agree on the ideas behind the RPL model and what is to be assessed in the process, this should improve the possibility of assessing that which is actually supposed to be assessed.

\section{Learning to put prior learning into words}

Usually, students are advised to connect theory to their own experiences when writing papers or working with cases. In this context, prior experiences have consequences for traditional students. One participant in the project explained that even though she did not know of any academic theories, she had come up with her own theories based on working-life experiences. After the assessment of the cases, one of the teachers came to the conclusion that traditional students' answers are often characterised by 'theorised practice', while these participants' answers, on the contrary, are characterised by 'practised theory'. That is, they already knew to some extent the theory from practice, even if they had not expressed it explicitly in written (or spoken) language before, or used academic concepts and terms.

What we became aware of is that the RPL process must be considered to be a learning process; but not only learning in the usual sense. Firstly, the participants had to re-learn what they already knew, i.e. learning to express their prior often tacit knowledge. But the participants also learned something new in the RPL process. The RPL process, in these initial courses, began an orientation in the specific 'language' of higher education. In other words, the process initiated the development of one aspect of graduateness, appropriating the academic language, which was not part of their prior learning. In this process, mutual understanding between assessor and assessee turned out to be important for integrating the participants into the higher education context. Peters (2005) also described this as learning the discourse of higher education something Peters saw as problematic since it could exclude individuals. However, these learning processes made the transfer and 'translation' of knowledge from working life to academia possible to some extent. These experiences may also have contributed to both an interest and a tool for further studying. 


\section{RPL in higher education for accreditation - some critical reflections}

Above, we reported on an RPL project designed to provide accreditation in higher education. Some of the key problems that occurred in the project were highlighted. We argued that mutual understanding between assessor and assessees was crucial in the RPL process. Nevertheless, we now also want to address some more general problems with RPL, based on this project, as regards accreditation. Here, we will use some aspects from Habermas' theory of communicative action to underpin a more critical review. One of the participants reported the following in the evaluation sheet sent out after the end of the RPL process:

"The absolutely best thing is that it has been connected to the credit system of the University. I have seen numerous RPL alternatives that result in nothing more than a certificate, and this is not valued to any great extent, in this 'point-setting' [the participant's own quotation marks] world in which we are working".

Even though the participant is in favour of the RPL process providing 'real' credits and not merely a certificate, the quote also presents a quite cynical view of knowledge. Is it really possible to give accreditation to experiential learning without losing something vital in the reason for attending higher education? What kind of view of knowledge does RPL for accreditation promote? RPL for accreditation could be seen as a rather instrumental form of assimilation of knowledge by the system (in this case, the higher education system). Here, only those experiences that fit the system are acknowledged. Habermas addresses this concern and states that: '[ . . ] autonomous subsystems make their way into the lifeworld from the outside like colonial masters coming into tribal society - and force a process of assimilation upon it' (Habermas 1987 p. 355). What is problematic here is that individual knowledge, of e.g. the trade union workers discussed in this article, is not fully addressed and many experiences will not be acknowledged by the system. Based on the experiences in the project addressed here, RPL for accreditation becomes a way for the system to value experiential learning and re-order it so it fits the curriculum.

Habermas considers education to be an important part of the reproduction of the lifeworld. Through education, we as individuals are socialised and from this socialisation our identities are constructed and developed. In the end, this process is part of a reproduction of society as a lifeworld. When RPL serves as a tool to assimilate experiences, this reproduction will not occur. Instead, experiences are assimilated (e.g. experiences are only acknowledged when they fit the demands of the system) and processes of mutual learning are excluded. Knowledge or prior learning then becomes something that can be used to trade and bargain for a grade in a course; in much the same way as the medium of money in the economy. There is thus a risk that language will become a subordinated medium in RPL processes, which instead function by means of an instrumental rationality. RPL for accreditation could thus be seen as a colonisation of the lifeworld. But if language could serve as a coordinating medium, and communicative rationality could underpin the RPL process, this could contribute to a more fair and valid assessment procedure, e.g. a procedure where mutual understanding between assessor and assessee is seen as fundamental. 


\section{Conclusion}

As discussed above, academic terms, learning in RPL, assessment and language proficiency became main issues in the development of an RPL model. The model was very much inspired by Habermas and the importance of mutual understanding. We also moved forward into a more critical discussion, based on Habermas. Here, RPL for accreditation was discussed as a problematic form of RPL, where prior learning is being used to exchange for a grade.

If we go back to the beginning of the project, many of the participants expressed the belief that higher education provided 'truths' exclusively. But RPL for accreditation is actually based on the assumption that learning from experiences from other contexts such as working life and leisure could be recognised and assessed according to specific criteria. So if RPL in this context is supposed to be possible, we must believe that experiences and knowledge gained in different settings and contexts via translation processes, such as RPL, can be proven to be comparable, i.e. fit each other. The argument we have tried to present is that assessors and assessees in RPL then must reach mutual understanding of the logic of the RPL model and the world of higher education. This also included a major effort in trying to reach a mutual definition of the ideas behind the RPL model - in Habermasian words, defining the situation (Habermas 1984; 1987). If RPL for accreditation progresses through mutual understanding, this could support fairness and enhance validity.

There are also more general problems with RPL for accreditation in higher education. RPL in this context seems to promote a rather technical and instrumental view of knowledge - as if knowledge could be used as 'money' to trade and exchange for a 'grade'. From a Habermasian perspective, this could be seen as a colonisation of the lifeworld.

It should also be of importance to address the question of what could be lost, when RPL for accreditation is being used. The RPL process studied here only included a few initial courses. Nevertheless, we could see how this process initiated the development of one aspect of 'graduateness' (Wheelahan 2006), i.e. the appropriation of the academic language. On the one hand, a more encompassing (more courses or a whole program) RPL process might suggest that the participants get credentials but have limited opportunities (compared to 'ordinary' students) to take part in conversations, discussions, group work etc. These are all learning experiences that contribute to the outcome and purpose of higher education in terms of graduateness, and limited access to such experiences could be problematic. On the other hand, RPL in higher education could be developed further, e.g. if it is seen as part of more formal learning processes. For instance, in Volbrecht's (2009) study of a teacher education initiative in South Africa (2009) a high degree of 'Trojan Horse RPL' (Harris 1999) where seen, where RPL as integrated part of the curriculum could bring new groups into the university. Drawing from Valk's (2009) suggestions in a study of higher education in the UK, RPL could take place in official university courses where candidates reflect on, identify and give proof of their prior learning. Thus, RPL could be an integrated part in courses and programs, which include and take into account the individual's prior experiential learning. But, then more as a starting point in courses and programs where the expected graduateness is developed as well.

Finally, the results of this article and the context on which these results are based show that there is a need for more critical research on RPL for accreditation in higher education. For example, there is a lack of research on the validity of RPL models. Stenlund (2009) has criticised RPL/APL for accreditation based on the lack of both theoretical and empirical research on the validity of RPL models. What is actually 
confirmed and recognised in such RPL processes and are they in fact valid? Our conclusion based on the present case is that validity could be strengthened when assessor and assessee develop a mutual understanding in the assessment and RPL process. However, this idea needs to be developed further.

\section{Note on contributors}

PER ANDERSSON is Associate Professor of Education at the Department of Behavioural Sciences and Learning, Linköping University, Sweden. His main research interest is educational assessment and, in particular, recognition of prior learning.

FREDRIK SANDBERG is a PhD student in Adult learning at the Department of Behavioural Sciences and Learning, Linköping University, Sweden. His dissertation project critically explores recognition of prior learning with a focus on RPL in general, but mainly on such processes in the health care sector.

\section{References}

Andersson, P., and J. Harris, eds. 2006. Re-theorising the recognition of prior learning. Leicester: NIACE.

Castle, J., and G. Attwood. 2001. Recognition of prior learning (RPL) for access or credit? Problematic issues in a university adult education department in South Africa. Studies in the Education of Adults 33, no. 1: 60-71.

Chisholm, C., and M. Davis. 2007. Analysis and evaluation of factors relating to accrediting $100 \%$ of prior experiential learning in UK work-based awards. Assessment \& Evaluation in Higher Education 32, no. 1: 45-59.

Dahlgren, L.O., A. Fejes, M. Abrandt-Dahlgren, and N. Trowald. 2009. Grading systems, features of assessment and students' approaches to learning. Teaching in Higher Education 14, no. 2: 185-94.

Habermas, J. 1984. The theory of communicative action, volume one: Reason and the rationalization of society. Cambridge: Polity Press.

Habermas, J. 1987. The theory of communicative action, volume two: The critique of functionalist reason. Cambridge: Polity Press.

Habermas, J. (1992). Postmetaphysical thinking. Cambridge : Polity Press, 1992

Harris, J. 1999. Ways of seeing the recognition of prior learning (RPL): What contribution can such practices make to social inclusion? Studies in the Education of Adults 31, no. 2: 124-39.

Husserl, E. (1970). The crisis of European sciences and transcendental phenomenology : an introduction to phenomenological philosophy. Evanston: Northwestern U.P.

Kirkland, M.C. 1971. The effects of tests on students and schools. Review of Educational Research 41, no. 4: 303-50.

Madaus, G.F. 1988. The influence of testing on the curriculum. In Critical issues in curriculum: Eighty-seventh yearbook of the National Society for the Study of Education. Part I, ed. L.N. Tanner, 83-121. Chicago, Illinois: The University of Chicago Press.

Miller, C.M.L., and M. Parlett. 1974. Up to the mark: A study of the examination game. London: Society for Research into Higher Education.

Orwell, G. 1950. 1984: a novel. New York: New American library.

Peters, H. 2005. Contested discourses: assessing the outcomes of learning from experience for the award of credit in higher education. Assessment \& Evaluation 
in Higher Education 30, no. 3: 273-85.

Pokorny, H. 2006. Recognising prior learning: What do we know? In Andersson and Harris 2006, 261-81.

Sandberg, F., and L. Schultz. 2007. Validus: Slutrapport för fas 1 i ett samverkansprojekt om validering av fackliga kunskaper inhämtade genom arbetsplatslärande [Validus: Final report from phase 1 of a cooperation project on recognition of work-based trade union knowledge]. Lund, Sweden: Lunds universitet.

Schultz, L., and F. Sandberg. 200 7. Validus II: Rapport från fas 2 av ett samverkansprojekt gällande validering av fackliga kunskaper inhämtade genom arbetsplatslärande [Validus II: Report from phase 2 of a cooperation project on recognition of work-based trade union knowledge]. Lund, Sweden: Lunds universitet.arbetsplatslärande. Lund, Sweden: Lunds universitet.

Snyder, B.R. 1970. The hidden curriculum. Cambridge, Massachusetts: The MIT Press.

Starr-Glass, D. 2002. Metaphor and totem: Exploring and evaluating prior experiential learning. Assessment \& Evaluation in Higher Education 27, no. 3: 221-31.

Starr-Glass, D., and A. Schwartzbaum. 2003. A liminal space: Challenges and opportunities in accreditation of prior learning in Judaic studies. Assessment \& Evaluation in Higher Education 28, no. 2: 179-92.

Stenlund, T. (2009). Assessment of prior learning in higher education: a review from a validity perspective. Assessment \& Evaluation in Higher Education. (iFirst) doi:10.1080/02602930902977798

Valk, A. 2009. Recognition of prior and experiential learning in European universities. Assessment in Education: Principles, Policy \& Practice 16, no. 1: 83-95.

Volbrecht, T. 2009. New courses for Trojan horses: rethinking RPL in a South African teacher education curriculum. Studies in Continuing Education 31, no. 1: $13-27$.

Warnke, G. 1987. Gadamer: Hermeneutics, tradition and reason. Cambridge: Polity Press.

Wheelahan, L. 2006. Vocations, 'graduateness' and the recognition of prior learning. In Andersson and Harris 2006, 241-60. 